\title{
Reasons to recommend lifestyle changes in type 2 diabetes mellitus and obesity
}

Rainer Spiegel

In her Research Highlight (Diabetes: Look AHEAD published: weight loss not linked to fewer cardiovascular events in patients with type 2 diabetes. Nat. Rev. Cardiol. 10, 429 [2013]), ${ }^{1}$ Bryony M. Mearns focuses on data from the randomized, multicentre Look AHEAD study, ${ }^{2}$ where intensive lifestyle modifications were not linked to fewer cardiovascular events during a 10 -year follow-up period in patients with type 2 diabetes mellitus and obesity. This finding has important implications in various clinical fields, including cardiology, endocrinology, and nephrology. Clinicians often suggest lifestyle modifications with the aim of reducing coronary heart disease. On the basis of the new evidence, the question arises whether lifestyle modifications, such as weight loss, should still be recommended in the absence of a demonstrated reduction in cardiovascular events. As a clinician with a particular interest in cardiology, endocrinology, and nephrology, I would still suggest lifestyle modifications for three reasons.

First, the reduction of cardiovascular events is not the only reason that clinicians suggest lifestyle modifications. An equally important reason is to prevent end-stage renal disease, which is linked to obesity, hypertension, and type 2 diabetes, ${ }^{3,4}$ and has not been addressed in the Look AHEAD study. ${ }^{2}$

Second, evidence demonstrates a link between a decrease in glomerular filtration rate and atherosclerosis. ${ }^{5-10}$ Given that decreased renal function is a risk factor for atherosclerosis, ${ }^{4-10}$ and also linked to type 2 diabetes and obesity, ${ }^{3,4}$ its neglect in the Look AHEAD study ${ }^{2}$ implies that not all risk factors have been controlled for. Investigators in future studies will need to incorporate renal function to judge whether a link exists between weight loss and cardiovascular events in patients with type 2 diabetes and obesity.

Third, the question arises as to why participants in the Look AHEAD study ${ }^{2}$ did not benefit from intensive lifestyle modifications in terms of cardiovascular events. One possible reason could be that, upon recruitment, atherosclerosis was already present in a large proportion of the study participants. The aim of lifestyle modifications, however, is to prevent premature onset of atherosclerosis, as this preventive step is likely to reduce the incidence of cardiovascular events. Therefore, starting lifestyle modifications at a much earlier stage than in the Look AHEAD study ${ }^{2}$ (where the youngest participants were aged 45 years, and the mean age was slightly $>58$ years) is vital. Modifications should optimally happen before diabetes and obesity develop. Healthy lifestyles could be encouraged at the community level, in schools, and by local health centres, with a focus on children and adolescents-that is, long before people are referred to a cardiologist for treatment.

Heidelberg University Hospital, Department of Internal Medicine I (Endocrinology, Nephrology, Osteology) and Clinical Chemistry, Im Neuenheimer Feld 410, 69210 Heidelberg, Germany.

rainer.spiegel@med.uni-heidelberg.de
Competing interests

The author declares no competing interests.

1. Mearns, B. M. Diabetes: Look AHEAD published: weight loss not linked to fewer cardiovascular events in patients with type 2 diabetes. Nat. Rev. Cardiol. 10, 429 (2013).

2. Look AHEAD Research Group. Cardiovascular effects of intensive lifestyle intervention in type 2 diabetes. N. Engl. J. Med. 369, 145-154 (2013).

3. Wang, Y. et al. Association between obesity and kidney disease: a systematic review and metaanalysis. Kidney Int. 73, 19-33 (2008).

4. Stenvinkel, P., Ikizler, T. A., Mallamaci, F. \& Zoccali, C. Obesity and nephrology: results of a knowledge and practice pattern survey. Nephrol. Dial. Transplant. http://dx.doi.org/ 10.1093/ndt/gft193.

5. Lu, B., Wan, J., Yang, Y., Li, Y. \& Hu, R The estimated glomerular filtration rate is associated with subclinical atherosclerosis, independently of albuminuria, in patients with type 2 diabetes. Int. Angiol. 32, 532-539 (2013).

6. Hui, X. et al. CKD and cardiovascular disease in the Atherosclerosis Risk In Communities (ARIC) study: interactions with age, sex, and race. Am. J. Kidney Dis. http://dx.doi.org/10.1053/ j.ajkd.2013.04.010.

7. Ito, H. et al. The prevalence of the risk factors for atherosclerosis among type 2 diabetic patients is greater in the progressive stages of chronic kidney disease. Nephron Extra 12, 66-72 (2013).

8. Yamashita, T. et al. Renal insufficiency without albuminuria is associated with peripheral artery atherosclerosis and lipid metabolism disorders in patients with type 2 diabetes. J. Atheroscler. Thromb. http://dx.doi.org/10.5551/ jat.15669.

9. Kokubo, Y. Carotid atherosclerosis in kidney disease. Contrib. Nephrol. 179, 35-41 (2013).

10. Olechnowicz-Tietz, S., Gluba, A., Paradowska, A., Banach, M. \& Rysz, J. The risk of atherosclerosis in patients with chronic kidney disease. Int. Urol. Nephrol. http:// dx.doi.org/10.1007/s11255-013-0407-1. 